\title{
IC5063: A MERGER WITH A HIDDEN LUMINOUS ACTIVE NUCLEUS
}

\author{
L. Colina*, W. Sparks, F. Macchetto* \\ Space Telescope Science Institute, Baltimore, U.S.A. \\ * Affiliated to the Astrophysics Division, ESTEC, Noordwijk, The Netherlands.
}

\section{INTRODUCTION}

Despite intense theoretical and observational effort during the past few years, two important questions in the field of active galaxies remain open and are the subject of vigorous debate.

The first concerns the connection between the interaction/merger of galaxies and the generation of starburst and/or active nuclei. A large fraction of high luminosity radio galaxies (Baum et al. 1988), quasars (Hutchings, 1987) and IRAS galaxies (Sanders et al. 1988), show the presence of a large companion or have a peculiar optical morphology which is offen interpreted as evidence of a recent merger or interaction. In Seyferts, there are also several examples of individual galaxies with clear evidence of interaction/merger (Colina et al. 1987; Fricke and Kollatschny, 1989; Macchetto et al. 1990). Also a significant fraction of low luminosity radio galaxies are associated with a system of two elliptical galaries in interaction (Colina and Pérez-Fournon, 1990a,b).

The second question concerns the anisotropy of the non-thermal nuclear source. A large body of evidence has been collected during the past five years showing that all types of active galaxies could radiate anisotropically (obscuration/beaming/accretion disc/ionization cones) and suggesting therefore that the divernity of AGN: can simply be explained by a different viewing angle (see Browne, 1989 for a review).

IC5063 is a nearby galaxy classified as an SO and containing a system of dust lanes parallel to its major optical axis (Danziger, Goss and Wellington, 1981; Bergeron, Durret and Boksenberg, 1983). Extended emission line regions with high excitation properties have been detected over distances of up to $19 \mathrm{kpc}$ from the nucleus. This galaxy has been classified as Seyfert 2 on the basis of its emission line spectrum. These characteristics make IC5063 one of the best candidates for a merger remnant and an excellent candidate for a hidden luminous active nucleus. Based on new broad and narrow band images and long-8lit spectroscopy obtained at the ESO $3.6 \mathrm{~m}$ telescope using EFOSC, we present some preliminary results supporting this hypothesis. 


\section{RESULTS AND DISCUSSION}

(a) Origin of the gas/dust: merger remnant

Fig.1a shows the image of IC5063 in the $B$ broad-band filter while Fig.1b presents an extinction map derived by ratioing the $B$ image to an elliptical model of the $R$ image (Sparks et al. 1985). This shows a complex system of dust lanes mostly in the northern half of IC5063. The morphology represents a zig-zagging distribution running roughly parallel to the major axis of IC5063 extending from very large radii all the way into the nucleus. This supports the idea that the dust has an external origin since such structures are unlikely to survive for long due to differential rotation effect. We are witnessing gas in the process of relaxing into the principal plane of the galaxy.

The distribution of the ionized gas is presented in Figure $1 \mathrm{c} \& \mathrm{~d}$. We see a main body with three radial filaments in position angles $285^{\circ}-290^{\circ}, 310^{\circ}-315^{\circ}$ and $325^{\circ}-330^{\circ}$. The size of these emission regions is $\approx 69^{\prime \prime}$ by $20^{\prime \prime}$ (i.e. 22 by $6.4 \mathrm{kpc}$ for $\mathrm{H}_{0}=50 \mathrm{~km} \mathrm{~s}^{-1} \mathrm{Mpc}^{-1}$ ) along the major and minor axis respectively. Also an extension of the PA285 filament is detected in [OIII] bending toward west at PA270 and at distances of up to $90^{\prime \prime}$ from the nucleus (i.e. $29 \mathrm{kpc}$ ).

The morphological characteristics, together with the kinematic properties and heavy element abundances of the gas suggest an external origin (Colina et al. 1990, in preparation).

\section{(b) Hidden Luminous Active Nucleus}

The high $\mathrm{S} / \mathrm{N}$ of our spectrum enables us to find evidence for the presence of a very powerful non-thermal source hidden in the nucleus of IC5063. First, we detect high excitation emission lines of $[\mathrm{FeVII}] \lambda 5721,6087$ (ionization potential $\mathrm{Fe}^{6+}=100 \mathrm{eV}$ ) and less clearly, $[\mathrm{CaV}] \lambda 5309$ and $[\mathrm{FeX}] \lambda 6375$. These lines are only detected within 1.5-2" around the nucleus (see Figure 2), indicating therefore the presence of a local and hard extreme ultraviolet ionizing source. We do not have any temperature indicator for the regions emitting these lines but in other Seyfert galaxies where these high excitation lines have been detected (i.e. Tol0109-383: Fosbury and Sansom 1983), the temperatures were consistent with photoionization.

Second, we detect a faint broad H $\alpha$ emission line. This component is also only observed within 1" of the nucleus (see Figures 3a,b). Its FWZI corresponds to a velocity of $6000 \mathrm{~km} \mathrm{~s}^{-1}$, which confirms the claim by Bergeron, Durret and Boksenberg (1983) based on deblending techniques. This faint broad emission component may arise in an obscured broad line region. 


\section{SUMMARY}

The results obtained using new deep optical images and long-slit spectroscopy of IC5063 and its associated extended emission line regions, can be summarised as follows:

(a) We detect a system of zig-zagging dust lanes over the whole galaxy, concentrated in the northern regions, and running approximely parallel to the major optical axis. We also detect high excitation extended emission line regions concentrated along the major axis of the galaxy and with a total size of $22 \times 6.4 \mathrm{kpc}$. This system of gas and dust with its peculiar morphology most likely has an external origin and can be considered as the likely remnant of a recent merger.

(b) The spectra of the nucleus of IC5063 shows high excitation lines like [FeVII] 6087 and a faint broad $\mathrm{H} \alpha$ emission line component (FWZI $\approx 6000 \mathrm{~km} \cdot \mathrm{s}^{-1}$ ) This is evidence for the presence of a hidden Seyfert 1 type nucleus located at the center of IC5063.

\section{REFERENCES}

Baum, S.A., Heckman, T.M., Bridle, A., van Breugel, W., and Miley, G., 1988, Ap. J. Suppl., 68, 526.

Bergeron, J., Durret, F., and Boksenberg, A., 1983, Astr. Ap., 127, 322.

Browne, I.W.A., 1989, ESO Workshop on Extranuclear Activity in Galaxies, Ed: E.J.A. Meurs and R.A.E. Bosbury, p. 379.

Colina, L., Fricke, K.J., Kollatschny, W., and Perryman, M.A.C. 1987, Astr. Ap., $178,51$.

Colina, L., and Pérez-Fournon, I., 1990a, Ap. J.Suppl., 72, 000.

Colina, L., and Pérez-Fournon, I., 1990b, Ap. J., 348, 000.

Danziger, I.J., Goss, W.M., and Wellington, K.J., 1981, M.N.R.A.S., 186, 845.

Fosbury, R.A.E., Sansom, A.E., 1983, M.N.R.A.S., 204, 1231.

Fricke, K.J., and Kollatschny, W., 1989, I.A.U. Symposium 134, Proc. Conf. Active Galactic Nuclei, ed: D.E. Osterbrock and J.S. Miller, (Dordrecht: Reidel), p.425.

Hutchings, J.B., 1987, Ap.J., 320, 122.

Macchetto, F.D., Colina, L., Golombek, D., Perryman, M.A.C., and di Serego Alighieri, 1990, Ap. J., , in press.

Sanders, D.B., Soifer, B.T., Elias, J.H., Madore, B.F., Matthews, K., Neugebauer, G., and Scoville, N.Z., 1988, Ap. J., 325, 74.

Sparks, W.B., Wall, J.V., Thorne, D., Jordan, P., van Breda, I.G., Rudd, P., Jorgensen, H.E., 1985, M.N.R.A.S., $217,87$. 


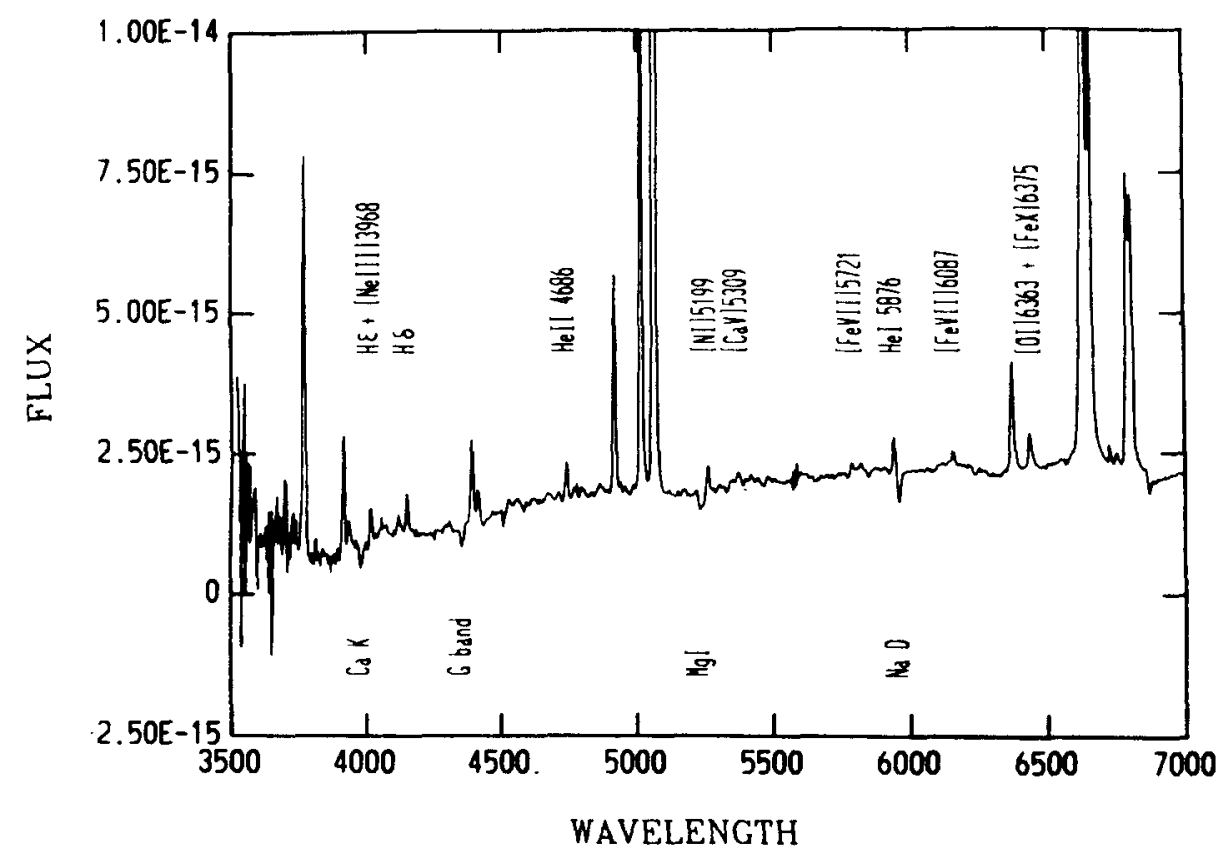

Figure 2: Spectrum of IC5063 nucleus showing all the strong emission lines and the faint high excitation lines.
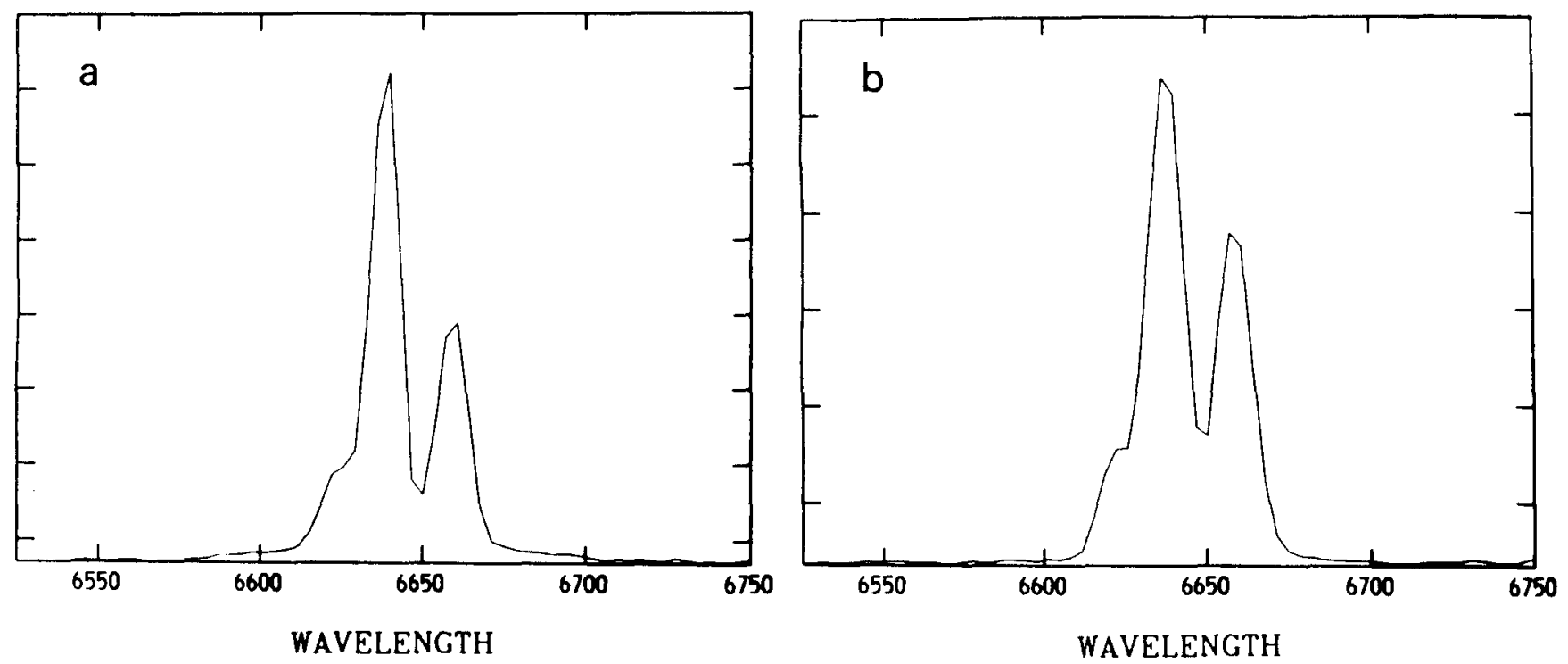

Figure 3: (a) Profile of the $\mathrm{H} \alpha$ line centered on the nucleus. The faint broad emission line component with FWZI $\approx 6000 \mathrm{~km} \cdot \mathrm{s}^{-1}$ is clearly visible. (b) The same emission line profile but centered at $1.4^{\prime \prime} \mathrm{SE}$ of the nucleus along PA289 ${ }^{\circ}$. Note that the broad component is no longer present. 


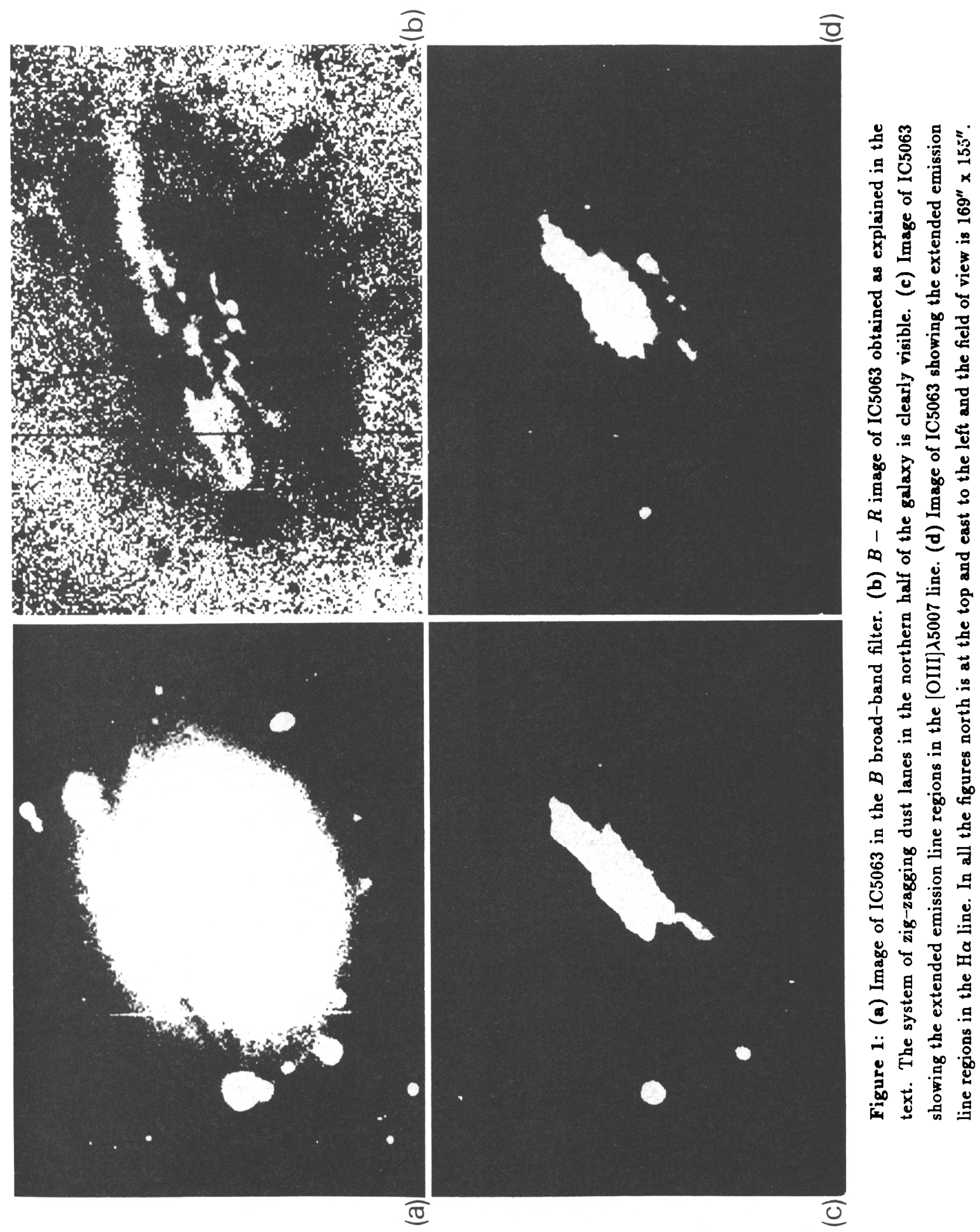

